\title{
ÓRGÃOS INTERNOS E TRATO DIGESTÓRIO DE NOVILHOS E VACAS DE DESCARTE DE DIFERENTES GRUPOS GENÉTICOS
}

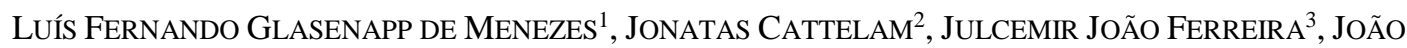 \\ RESTLE $^{4}$, IVAN LUIZ BRONDANI ${ }^{5}$, DARI CELESTINO ALVES FILHO ${ }^{5}$ \\ 1 Professor Doutor da Universidade Tecnológica Federal do Paraná, Dois Vizinhos, PR, Brasil. \\ luismenezes@utfpr.edu.br \\ 2 Mestre, Pós-graduando da Universidade Federal de Santa Maria, Santa Maria, RS, Brasil \\ 3 Zootecnista, Mestre, Bunge Fertilizantes, Ponta Grossa, PR, Brasil \\ 4 Professor Visitante Nacional Senior (CAPES) na Universidade Federal do Tocantins, Araguaína, TO, Brasil \\ 5 Professores Doutores da Universidade Federal de Santa Maria, Santa Maria, RS, Brasil
}

\begin{abstract}
O objetivo deste estudo foi avaliar os órgãos internos e o trato digestório de novilhos e vacas de descarte 5/8 Charolês (Ch) $3 / 8$ Nelore $(\mathrm{Ne})$ e $5 / 8 \mathrm{Ne} 3 / 8 \mathrm{Ch}$, terminados em confinamento. Foram utilizados 12 novilhos e 12 vacas de descarte, sendo cada categoria composta por seis animais 5/8Ch $3 / 8 \mathrm{Ne}$ e seis animais $5 / 8 \mathrm{Ne} 3 / 8 \mathrm{Ch}$. A idade média dos animais ao final do período experimental foi de 23 meses para os novilhos e 68 meses para as vacas de descarte. Os animais foram terminados em confinamento até atingirem acabamento para o abate. A dieta alimentar continha relação volumoso:concentrado de 40:60 (base na matéria seca). O delineamento experimental foi $\mathrm{o}$ inteiramente casualizado, em um arranjo fatorial $2 \times 2$ (2 categorias x 2 grupos genéticos. Os dados foram submetidos à análise de variância pelo proc GLM, sendo as médias classificadas pelo teste " $F$ " e os parâmetros com efeito significativo para interação entre categoria e grupo genético comparados pelo "teste t", a 5\% de significância. Houve maior peso de abate (PAB) $(509,67$ contra 414,50 $\mathrm{kg}$ ) e de corpo vazio (PCVZ) $(433,01$ contra $355,27 \mathrm{~kg}$,
\end{abstract}

respectivamente) para as vacas em relação aos novilhos. As vacas apresentaram maiores pesos absolutos para coração $(1,62$ contra $1,40 \mathrm{~kg})$, fígado $(7,07$ contra $5,95 \mathrm{~kg})$ e também para o conjunto de órgãos internos, total do trato digestório e sangue. Quando os pesos dos componentes foram expressos em porcentagem do PCVZ, os novilhos foram superiores no peso dos pulmões $(1,08$ contra $0,95 \%)$, baço $(0,29$ contra $0,22 \%)$, intestinos $(2,04$ contra $1,72 \%)$ e no total dos órgãos vitais (3,92 contra $3,63 \%$ ). Quanto aos grupos genéticos, animais 5/8Ch $3 / 8 \mathrm{Ne}$ apresentaram maior peso absoluto de trato digestório. Não houve correlação entre o rendimento de carcaça com componentes não integrantes da carcaça expressos em peso absoluto. Vacas de descarte apresentam maior massa dos órgãos internos e do trato digestório que novilhos abatidos aos 24 meses, enquanto bovinos do grupo genético $5 / 8 \mathrm{Ch} 3 / 8 \mathrm{Ne}$ apresentam maior peso de compartimentos e do total do trato digestório em relação a bovinos $5 / 8 \mathrm{Ne} 3 / 8 \mathrm{Ch}$.

PALAVRAS-CHAVE: Abomaso; categoria animal; fígado; peso de corpo vazio; rendimento de carcaça..

\section{INTERNAL ORGANS AND DIGESTIVE TRACT FROM STEERS AND CULL COWS OF DIFFERENT GENETIC GROUPS}

\section{ABSTRACT}

The objective of this study was to evaluate the internal organs and digestive tract of two categories, steers and cull cows, from two genetic groups: 5/8 Charolais $(\mathrm{Ch})$ $3 / 8$ Nellore $(\mathrm{Ne})$ and $5 / 8 \mathrm{Ne} 3 / 8 \mathrm{Ch}$, finished in feedlot. We used 12 steers and 12 cull cows, being the categories compounded for six $5 / 8 \mathrm{Ch} 3 / 8 \mathrm{Ne}$ animals and six animals $5 / 8 \mathrm{Ne} 3 / 8 \mathrm{Ch}$. The average age of animals at the end of the experimental period was 23 and 68 months for steers and cull cows, respectively. The animals were feedlot-finished until reaching finishing degree for slaughter. The 
experimental diet had a roughage:concentrate ratio of 40:60 (dry matter basis). The experimental design was completely randomized in a $2 \times 2$ (two categories and two genetic groups) factorial scheme. Data were submitted to analysis of variance by proc GLM, being the means classified by test " $F$ " and the parameters with significant effects for interaction between genetic group and category compared by "test $\mathrm{t}$ ", with 5\% significance level. Slaughter weight (SW) (509.67 versus $414.50 \mathrm{~kg})$ and empty body weight (EBW) (433.01 versus $355.27 \mathrm{~kg}$, respectively) were higher for cows in relation to steers. The cull cows showed higher absolute weight for heart (1.62 versus $1.40 \mathrm{~kg})$, liver (7.07 versus $5.95 \mathrm{~kg}$ ) and for total internal organs, digestive tract and blood. When the components were adjusted for percentage of EBW the steers were superiors on lungs (1.08 versus $0.95 \%)$, spleen $(0.29$ versus $0.22 \%)$ intestines $(2.04$ versus $1.70 \%)$ and total internal organs weight (3.91 versus $3.60 \%)$. As genetic groups, $5 / 8 \mathrm{Ch} 3 / 8 \mathrm{Ne}$ animals showed higher absolute weight for digestive tract. The carcass dressing percentage and non-carcass components, in absolute weight, showed correlation. Cull cows showed higher weight for internal organs and digestive tract than steers slaughtered at 24 months, while cattle for genetic group $5 / 8 \mathrm{Ch} 3 / 8 \mathrm{Ne}$ showed higher weight for compartments and total of digestive tract in relation to $5 / 8 \mathrm{Ne} 3 / 8 \mathrm{Ch}$ bovines.

KEYWORDS: abomasum; animal category; carcass dressing percentage; empty body weight; liver.

\section{INTRODUÇÃO}

O Brasil vem nos últimos anos ocupando lugar de destaque no mercado internacional pela alta quantidade de carne bovina produzida e exportada. Neste sentido, buscam-se aumentar os índices de produtividade e minimizar os custos de produção para aumentar a receita.

$\mathrm{O}$ abate de vacas de descarte representou 46\% do total em 2010 (ANUALPEC, 2010), sendo, das categorias utilizadas para a produção, a que mais cresceu. Esse aumento deve-se a vários fatores: incremento tecnológico nas propriedades, melhorando fatores relacionados à nutrição, melhoramento genético e técnicas de manejo; aumento nas exportações de carne bovina; e acabamento mais rápido das vacas de descarte por serem animais adultos, diminuindo o custo de produção.

Além disso, a variedade genética dos bovinos abatidos no território brasileiro é bastante grande. A raça mais criada no Brasil é a Nelore, que se caracteriza por apresentar maior rendimento de carcaça do que outros genótipos criados nas mesmas condições. A raça Charolês está entre as raças mais difundidas na região Sul do Brasil, principalmente por apresentar carcaças pesadas e com grande musculosidade. Entretanto, existem poucos estudos relacionando os componentes não integrantes da carcaça e o rendimento de carcaça, principalmente levando em consideração vacas de descarte, que já chegam a quase $50 \%$ dos animais abatidos no Brasil (ANUALPEC, 2010).

As partes não-integrantes da carcaça, também denominadas de componentes de corpo vazio, apresentam influência direta sobre $o$ rendimento da carcaça dos bovinos (OWENS et al, 1995). Conforme VAZ et al. (2010), fêmeas apresentam menor rendimento de carcaça que machos em virtude do maior peso relativo do couro, rúmen vazio, conteúdo do trato digestório e gordura depositada no úbere. As diferenças nas exigências de mantença podem estar associadas ao tamanho relativo dos órgãos, que são maiores em animais taurinos que em zebuínos (SILVA et al., 2002), ao estádio de maturidade, à raça e ao nível nutricional (GESUALDI JÚNIOR et al., 2001). JORGE \& FONTES (2001) ressaltam que animais de raças europeias ou mestiços de raças leiteiras tendem a apresentar maior massa de órgãos internos em relação ao peso vivo que animais zebuínos. De acordo com CATTON \& DHUYVETTER (1997), os tecidos viscerais consomem cerca de $50 \%$ da energia destinada para mantença.

Os componentes de corpo vazio são utilizados para a geração de receita nos frigoríficos. RIBEIRO et al. (2001) ressaltam que órgãos internos e componentes externos, por meio de processamento adequado, podem se tornar subprodutos valiosos da indústria da carne. Dessa forma, objetivou-se com este estudo quantificar os componentes do corpo não integrantes da carcaça de novilhos e vacas dos grupos genéticos 5/8 Charolês 3/8 Nelore e 5/8 Nelore 3/8 Charolês, terminados em confinamento.

\section{MATERIAL E MÉTODOS}

O experimento foi desenvolvido no Laboratório de Bovinocultura de Corte do Departamento de Zootecnia da Universidade Federal de Santa Maria, localizada no município de Santa Maria, Rio Grande do Sul.

Foram utilizados 24 animais, 12 novilhos e 12 vacas de descarte, sendo cada categoria composta por seis animais 5/8 Charolês $3 / 8$ Nelore $(5 / 8 \mathrm{Ch}$ $3 / 8 \mathrm{Ne}$ ) e seis animais $5 / 8$ Nelore $3 / 8$ Charolês $(5 / 8 \mathrm{Ne} 3 / 8 \mathrm{Ch})$, todos oriundos de mesmo rebanho, com manejo sanitário e nutricional conjunto para 
cada categoria. O período de terminação dos animais ocorreu em confinamento por 99 dias para todos os animais, sendo que, ao início desse período, os novilhos apresentavam, em média, 21 meses e 272 $\mathrm{kg}$, enquanto as vacas apresentavam, em média, 66 meses e $340 \mathrm{~kg}$. A dieta foi à base de silagem de milho - AG 2020 nos primeiros 48 dias e AG 5011 no período restante (51 dias) - e concentrado composto de farelo de trigo, grão de milho moído, farelo de soja, calcário calcítico e sal, com relação volumoso:concentrado de 40:60 (base na matéria seca). A dieta com a silagem AG2020 continha $14,4 \%$ proteína bruta $(\mathrm{PB})$ e $2.784 \mathrm{kcal}$ de energia digestível (ED) e aquela com a silagem de milho AG5011, 14,00\% proteína bruta e $2.953 \mathrm{kcal}$ de energia digestível. A dieta foi formulada com o objetivo de atender a capacidade de consumo e exigência dos animais para ganho de peso médio diário de $1,70 \mathrm{~kg}$ (NRC, 2001).

O momento de abate foi determinado pelo critério de condição corporal, preconizando adequada composição de gordura de cobertura da carcaça, seguindo metodologia descrita por LOWAN (1973), em que escore corporal = 1 - muito magro; escore corporal $=5-$ muito gordo. Antes da condução ao frigorífico comercial, os animais foram submetidos a jejum de sólidos por 14 horas. Durante os procedimentos de frigorífico, o corpo de cada animal foi separado em cabeça, patas, orelhas, chifres (quando presentes), vassoura da cauda, couro (conjunto dos componentes externos); pulmão, fígado, rins, coração, baço (conjunto dos órgãos vitais); gordura de toalete, gordura inguinal/úbere (para machos e fêmeas, respectivamente), gordura renal, gordura ruminal, gordura do coração, gordura intestinal, gordura de abomaso (conjunto de gorduras internas); rúmen-retículo, omaso, abomaso, intestinos delgado e grosso (conjunto do trato digestório); sangue e aparelho reprodutivo. As duas meia-carcaças de cada animal foram pesadas antes do resfriamento, para obtenção do peso de carcaça quente, e após o resfriamento por 24 horas na câmara fria, para obtenção do peso de carcaça fria. $\mathrm{O}$ peso do corpo vazio foi obtido pela equação:

$k g P C V Z=p c q+k g c o n j e x t+k g o r g v i t a i s+k g g o r d u r a s+k g t d i g v z+k g$ sangue+kgaprep

em que pcq=peso de carcaça quente; $\mathrm{kgconjext=peso}$ do conjunto dos componentes externos; kgorgvitais=peso do conjunto dos órgãos vitais; kggorduras=peso do conjunto das gorduras internas; kgtdigvz=peso do trato digestivo vazio; kgsangue $=$ peso de sangue; kgaprep $=$ peso do aparelho reprodutivo.

$\mathrm{O}$ delineamento experimental foi o inteiramente casualizado em um arranjo fatorial $2 \times 2$ ( 2 categorias - novilhos e vacas $\mathrm{x} 2$ grupos genéticos - $5 / 8 \mathrm{Ch} 3 / 8 \mathrm{Ne}$ e $5 / 8 \mathrm{Ne} 3 / 8 \mathrm{Ch}$ ). As médias dos parâmetros em estudo foram submetidas à análise de variância. $\mathrm{O}$ modelo estatístico adotado foi:

$$
Y i j=\mu+C_{i}+G_{j}+\left(C^{*} G G\right)_{i j}+E_{i j k}
$$

em que $\mathrm{Y}_{\mathrm{ij}}=$ variáveis dependentes; $\mu=$ média de todas as observações; $\mathrm{C}_{\mathrm{i}}=$ efeito da categoria de ordem i $\left(1=\right.$ novilho e 2 = vaca) $\mathrm{GG}_{\mathrm{j}}=$ efeito do grupo genético de ordem $\mathrm{j}(1=5 / 8 \mathrm{Ch} 3 / 8 \mathrm{Ne}$ e $2=$ $5 / 8 \mathrm{Ne} 3 / 8 \mathrm{Ch}) ;\left(\mathrm{C}^{*} \mathrm{GG}\right)_{\mathrm{ij}}=$ efeito da interação da categoria $\mathrm{i}$ com o grupo genético $\mathrm{j} ; \mathrm{E}_{\mathrm{ijk}}=$ efeito residual aleatório. Para os efeitos principais, as médias foram classificadas pelo teste "F". Para os parâmetros com efeito significativo $(\mathrm{P}<0,05)$ para a interação $\mathrm{C}^{*} \mathrm{GG}$, as médias foram classificadas pelo "teste t" $(\mathrm{P}<0,05)$. Foram realizadas análises de correlação de Pearson pelo procedimento proc CORR entre as características estudadas. As análises estatísticas foram realizadas utilizando-se o programa estatístico SAS (2001).

\section{RESULTADOS E DISCUSSÃO}

As vacas apresentaram maior $(\mathrm{P}<0,05)$ peso de corpo vazio (Tabela 1), o que deve estar relacionado ao maior peso de abate das fêmeas, visto que essas variáveis são altamente correlacionadas. No presente estudo, a correlação entre essas duas variáveis foi significativa, $\operatorname{com} \mathrm{r}=0,99(\mathrm{P}<0,0001)$.

Para a relação entre os pesos de corpo vazio e de abate, houve diferença $(\mathrm{P}<0,05)$ entre as categorias de bovinos estudados e também entre os grupos genéticos. Segundo OWENS et al. (1995), várias equações de regressão foram descritas para estimar o peso corporal vazio por meio do peso vivo, sendo que o peso de corpo vazio correspondia de $85 \mathrm{a}$ 95\% do peso corporal. No presente estudo, essa relação foi de $85,57 \%$ para os novilhos e $84,15 \%$ para as vacas. Essa inferioridade das vacas pode demonstrar que o tempo de jejum semelhante para as duas categorias não é adequado, pois as vacas precisariam de um pouco mais de tempo para se igualar na perda do conteúdo do trato gastrintestinal. Diferença na relação entre os pesos de corpo vazio e de abate entre bovinos de diferentes categorias foi relatada por KUSS et al. (2009), que verificaram maior relação entre esses pesos em animais da categoria superjovem, abatidos com 16 meses de idade, em relação a novilhos jovens, abatidos com 26 meses de idade, com rendimentos de 92,39 e $89,76 \%$, respectivamente. Entre os grupos genéticos estudados, os bovinos com predomínio genético zebuíno apresentaram maior $(\mathrm{P}<0,05)$ relação entre os pesos de corpo vazio e de abate. MENEZES et al. (2007) observaram que novilhos de quarta geração 
com predomínio racial Nelore apresentaram maior rendimento de corpo vazio em relação a novilhos de predominância Charolês.

Quando os pesos dos rins (Tabela 2) foram expressos em valores absolutos, verificou-se interação significativa $(\mathrm{P}<0,05)$ entre categoria e grupo genético, de modo que as vacas $5 / 8 \mathrm{Ch} 3 / 8 \mathrm{Ne}$ apresentaram maiores pesos, enquanto que nos novilhos não houve diferença entre grupos genéticos.
Nas demais formas de expressão não houve diferença entre as categorias, mostrando que esse órgão mantém sua integridade, mesmo com o aumento da demanda de nutrientes. GESUALDI JÚNIOR et al. (2001) e SILVA et al. (2002) não observaram influência do nível de concentrado na dieta sobre o peso absoluto e relativo dos rins de bovinos.

Tabela 1 - Pesos de abate e de corpo vazio e relação entre os pesos de abate e de corpo vazio de acordo com a categoria e o grupo genético

\begin{tabular}{|c|c|c|c|}
\hline \multirow{2}{*}{ Grupo Genético } & \multicolumn{2}{|c|}{ Categoria } & \multirow{2}{*}{ Média } \\
\hline & Novilhos & Vacas & \\
\hline & \multicolumn{2}{|c|}{ Peso de abate, $\mathrm{kg}$} & \\
\hline $5 / 8 \mathrm{Ch} 3 / 8 \mathrm{Ne}$ & $417,67 \pm 21,28$ & $547,33 \pm 21,28$ & $482,50 \pm 15,05$ \\
\hline $5 / 8 \mathrm{Ne} 3 / 8 \mathrm{Ch}$ & $411,33 \pm 21,28$ & $472,00 \pm 21,28$ & $441,67 \pm 15,05$ \\
\hline \multirow[t]{2}{*}{ Média } & $414,50 \pm 15,05 \mathrm{D}$ & $509,67 \pm 15,05 \mathrm{C}$ & \\
\hline & \multicolumn{2}{|c|}{ Peso de corpo vazio, kg } & \\
\hline $5 / 8 \mathrm{Ch} 3 / 8 \mathrm{Ne}$ & $353,57 \pm 17,90$ & $463,48 \pm 17,90$ & $408,53 \pm 12,66$ \\
\hline $5 / 8 \mathrm{Ne} 3 / 8 \mathrm{Ch}$ & $356,97 \pm 17,90$ & $402,54 \pm 17,90$ & $379,80 \pm 12,66$ \\
\hline \multirow[t]{2}{*}{ Média } & $355,27 \pm 12,66 \mathrm{D}$ & $433,01 \pm 12,66 \mathrm{C}$ & \\
\hline & \multicolumn{2}{|c|}{ Relação peso de corpo vazio/ peso de abate, $\%$} & \\
\hline $5 / 8 \mathrm{Ch} 3 / 8 \mathrm{Ne}$ & $84,40 \pm 0,62$ & $83,88 \pm 0,62$ & $84,14 \pm 0,44 \mathrm{~B}$ \\
\hline $5 / 8 \mathrm{Ne} 3 / 8 \mathrm{Ch}$ & $86,75 \pm 0,62$ & $84,42 \pm 0,62$ & $85,58 \pm 0,44 \mathrm{~A}$ \\
\hline Média & $85,57 \pm 0,44 \mathrm{C}$ & $84,15 \pm 0,44 \mathrm{D}$ & \\
\hline
\end{tabular}

As vacas apresentaram maiores $(\mathrm{P}<0,05)$ pesos para coração e fígado quando expressos em valores absolutos (Tabela 2), possivelmente influenciados pela maior taxa metabólica desses animais em razão da sua alta taxa de ganho diário de peso $(1,60 \mathrm{~kg})$, causando recuperação da atividade metabólica do fígado (HOGG, 1991). PACHECO et al. (2005), ao avaliarem as características das partes não-integrantes da carcaça de novilhos do mesmo grupo genético que os do presente estudo, das categorias jovem, abatidos com 22 meses de idade, ou superjovem, abatidos aos 15 meses de idade, verificaram que os bovinos abatidos com idade mais avançada apresentaram maior peso absoluto de fígado, fato que os autores associaram à manifestação do ganho compensatório. Conforme JORGE \& FONTES (2001), o crescimento do fígado frente ao aumento da maturidade dos animais acompanha o crescimento do peso corporal vazio.

Do conjunto de órgãos vitais, FERREL \& JENKINS (1998) citam que o fígado é o que apresenta maiores variações diante de alterações no consumo de alimentos e níveis energéticos da dieta, devido à sua participação no metabolismo dos nutrientes. De acordo com VAN SOEST (1994), o fígado participa ativamente dos metabolismos energéticos e proteicos, com captação de cerca de $80 \%$ do propionato que passa pelo sistema portal para conversão em glicose. Segundo JOHNSON et al. (1990), o tamanho do fígado responde rapidamente às mudanças de consumo alimentar, apresentando desenvolvimento linear em resposta ao consumo de energia metabolizável.

Quando foram comparados os grupos genéticos, não foi verificada diferença para nenhum dos parâmetros avaliados na tabela 2, concordando com os resultados de MENEZES et al. (2007), que estudaram novilhos, e com os de KUSS et al. (2007), que estudaram vacas, todos utilizando animais do mesmo grupo genético do presente estudo.

Para o baço e os pulmões, foi observada diferença $(\mathrm{P}<0,05)$ entre as categorias, quando expressos em valores relativos a $100 \mathrm{~kg}$ do peso de abate, com superioridade para os novilhos. O menor peso dos órgãos acima citados nas fêmeas de descarte pode estar associado à maior pressão que esses órgãos sofrem, principalmente pelo aumento da deposição de gordura interna, conforme demonstrado por CATTELAN et al. (2010), e ao maior volume do trato gastrintestinal com o aumento do peso das 
vacas (Tabela 4).

Apesar do maior peso de abate das vacas, não houve diferença nos pesos absoluto e relativo do rúmen-retículo (Tabela 3). Similaridade no peso relativo do conjunto rúmen-retículo em bovinos de diferentes idades é reportada por PACHECO et al. (2005), para animais abatidos com 14 ou 24 meses de idade. A semelhança nos pesos absolutos do rúmen-retículo entre os grupos genéticos também corrobora os resultados encontrados por MENEZES et al. (2007), trabalhando com os mesmos grupos genéticos estudados. Por outro lado, esses autores observaram que, quando o peso do rúmen-retículo foi ajustado para o peso de corpo vazio, houve superioridade $(\mathrm{P}<0,10)$ dos animais $5 / 8 \mathrm{Ch} 3 / 8 \mathrm{Ne}$, diferente do encontrado no presente estudo.

Tabela 2 - Órgãos internos em peso absoluto e em relação aos pesos de corpo vazio (PCVZ) e de abate (PAB), de acordo com a categoria e grupo genético

\begin{tabular}{|c|c|c|c|c|c|c|c|c|c|}
\hline \multirow{2}{*}{ Grupo genético } & \multicolumn{2}{|c|}{ Categoria } & \multirow{2}{*}{ Média } & \multicolumn{2}{|c|}{ Categoria } & \multirow{2}{*}{ Média } & \multicolumn{2}{|c|}{ Categoria } & \multirow{2}{*}{ Média } \\
\hline & Novilho & Vaca & & Novilho & Vaca & & Novilho & Vaca & \\
\hline & \multicolumn{2}{|c|}{ Coração, kg } & & \multicolumn{2}{|c|}{ Coração, \% PCVZ } & & \multicolumn{2}{|c|}{ Coração, \% PAB } & \\
\hline $5 / 8 \mathrm{Ch} 3 / 8 \mathrm{Ne}$ & $1,41 \pm 0,10$ & $1,67 \pm 0,10$ & $1,54 \pm 0,07$ & $0,39 \pm 0,01$ & $0,36 \pm 0,01$ & $0,38 \pm 0,01$ & $0,33 \pm 0,01$ & $0,31 \pm 0,01$ & $0,32 \pm 0,01$ \\
\hline $5 / 8 \mathrm{Ne} 3 / 8 \mathrm{Ch}$ & $1,40 \pm 0,10$ & $1,57 \pm 0,10$ & $1,48 \pm 0,07$ & $0,39 \pm 0,01$ & $0,39 \pm 0,01$ & $0,39 \pm 0,01$ & $0,34 \pm 0,01$ & $0,33 \pm 0,01$ & $0,34 \pm 0,01$ \\
\hline \multirow[t]{2}{*}{ Média } & $1,40 \pm 0,07 \mathrm{D}$ & $1,62 \pm 0,07 \mathrm{C}$ & & $0,39 \pm 0,01$ & $0,38 \pm 0,01$ & & $0,34 \pm 0,01$ & $0,32 \pm 0,01$ & \\
\hline & \multicolumn{2}{|c|}{ Rins, $\mathrm{kg}$} & & \multicolumn{2}{|c|}{ Rins, $\%$ PCVZ } & & \multicolumn{2}{|c|}{ Rins, $\%$ PAB } & \\
\hline $5 / 8 \mathrm{Ch} 3 / 8 \mathrm{Ne}$ & $0,78 \pm 0,08 \mathrm{ab}$ & $0,98 \pm 0,08 \mathrm{a}$ & $0,88 \pm 0,06$ & $0,22 \pm 0,02$ & $0,22 \pm 0,02$ & $0,22 \pm 0,01$ & $0,19 \pm 0,02$ & $0,18 \pm 0,02$ & $0,18 \pm 0,01$ \\
\hline $5 / 8 \mathrm{Ne} 3 / 8 \mathrm{Ch}$ & $0,89 \pm 0,08 \mathrm{ab}$ & $0,74 \pm 0,08 \mathrm{~b}$ & $0,81 \pm 0,06$ & $0,25 \pm 0,02$ & $0,18 \pm 0,02$ & $0,22 \pm 0,01$ & $0,22 \pm 0,02$ & $0,16 \pm 0,02$ & $0,19 \pm 0,01$ \\
\hline \multirow{2}{*}{ Média } & $0,83 \pm 0,06$ & $0,86 \pm 0,06$ & & $0,23 \pm 0,01$ & $0,20 \pm 0,01$ & & $0,20 \pm 0,01$ & $0,17 \pm 0,01$ & \\
\hline & \multicolumn{2}{|c|}{ Pulmões, $\mathrm{kg}$} & & \multicolumn{2}{|c|}{ Pulmões, \% PCVZ } & & \multicolumn{2}{|c|}{ Pulmões, \% PAB } & \\
\hline $5 / 8 \mathrm{Ch} 3 / 8 \mathrm{Ne}$ & $4,39 \pm 0,34$ & $4,90 \pm 0,34$ & $4,64 \pm 0,24$ & $1,25 \pm 0,06$ & $1,07 \pm 0,06$ & $1,16 \pm 0,04$ & $1,05 \pm 0,05$ & $0,90 \pm 0,05$ & $0,98 \pm 0,03$ \\
\hline $5 / 8 \mathrm{Ne} 3 / 8 \mathrm{Ch}$ & $4,51 \pm 0,34$ & $4,78 \pm 0,34$ & $4,64 \pm 0,24$ & $1,26 \pm 0,06$ & $1,19 \pm 0,06$ & $1,22 \pm 0,04$ & $1,10 \pm 0,05$ & $1,00 \pm 0,05$ & $1,05 \pm 0,03$ \\
\hline \multirow[t]{2}{*}{ Média } & $4,44 \pm 0,24$ & $4,84 \pm 0,24$ & & $1,25 \pm 0,04$ & $1,13 \pm 0,04$ & & $1,08 \pm 0,03 \mathrm{~A}$ & $0,95 \pm 0,03 \mathrm{~B}$ & \\
\hline & \multicolumn{2}{|c|}{ Fígado, $\mathrm{kg}$} & & \multicolumn{2}{|c|}{ Fígado, \% PCVZ } & & \multicolumn{2}{|c|}{ Fígado, \% PAB } & \\
\hline $5 / 8 \mathrm{Ch} 3 / 8 \mathrm{Ne}$ & $5,96 \pm 0,31$ & $7,52 \pm 0,31$ & $6,74 \pm 0,21$ & $1,70 \pm 0,08$ & $1,64 \pm 0,08$ & $1,67 \pm 0,06$ & $1,44 \pm 0,06$ & $1,38 \pm 0,06$ & $1,41 \pm 0,05$ \\
\hline $5 / 8 \mathrm{Ne} 3 / 8 \mathrm{Ch}$ & $5,96 \pm 0,31$ & $6,62 \pm 0,31$ & $6,29 \pm 0,21$ & $1,68 \pm 0,08$ & $1,66 \pm 0,08$ & $1,67 \pm 0,06$ & $1,45 \pm 0,06$ & $1,41 \pm 0,06$ & $1,43 \pm 0,05$ \\
\hline \multirow[t]{2}{*}{ Média } & $5,95 \pm 0,21 \mathrm{D}$ & $7,07 \pm 0,21 \mathrm{C}$ & & $1,69 \pm 0,06$ & $1,66 \pm 0,06$ & & $1,44 \pm 0,05$ & $1,40 \pm 0,05$ & \\
\hline & \multicolumn{2}{|c|}{ Baço, kg } & & \multicolumn{2}{|c|}{ Baço, \% PCVZ } & & \multicolumn{2}{|c|}{ Baço, $\%$ PAB } & \\
\hline $5 / 8 \mathrm{Ch} 3 / 8 \mathrm{Ne}$ & $1,34 \pm 0,14$ & $1,05 \pm 0,14$ & $1,19 \pm 0,10$ & $0,38 \pm 0,04$ & $0,23 \pm 0,04$ & $0,30 \pm 0,03$ & $0,32 \pm 0,03$ & $0,19 \pm 0,03$ & $0,26 \pm 0,02$ \\
\hline $5 / 8 \mathrm{Ne} 3 / 8 \mathrm{Ch}$ & $1,12 \pm 0,14$ & $1,18 \pm 0,14$ & $1,15 \pm 0,10$ & $0,31 \pm 0,04$ & $0,30 \pm 0,04$ & $0,31 \pm 0,03$ & $0,27 \pm 0,03$ & $0,26 \pm 0,03$ & $0,26 \pm 0,02$ \\
\hline Média & $1,22 \pm 0,10$ & $1,11 \pm 0,10$ & & $0,34 \pm 0,02$ & $0,27 \pm 0,02$ & & $0,29 \pm 0,02 \mathrm{C}$ & $0,22 \pm 0,02 \mathrm{D}$ & \\
\hline
\end{tabular}

Tabela 3 - Trato digestório em peso absoluto e em relação aos pesos de corpo vazio (PCVZ) e de abate (PAB) de acordo com a categoria e grupo genético

\begin{tabular}{|c|c|c|c|c|c|c|c|c|c|}
\hline \multirow{3}{*}{ Grupo genético } & \multicolumn{2}{|c|}{ Categoria } & \multirow{3}{*}{ Média } & \multicolumn{2}{|c|}{ Categoria } & \multirow{3}{*}{ Média } & \multicolumn{2}{|c|}{ Categoria } & \multirow{3}{*}{ Média } \\
\hline & Novilho & Vaca & & Novilho & Vaca & & Novilho & Vaca & \\
\hline & \multicolumn{2}{|c|}{ Rúmen + retículo, kg } & & \multicolumn{2}{|c|}{ Rúmen + retículo, \% PCVZ } & & \multicolumn{2}{|c|}{ Rúmen + retículo,\% $\mathrm{PAB}$} & \\
\hline $5 / 8 \mathrm{Ch} 3 / 8 \mathrm{Ne}$ & $6,53 \pm 0,37 b$ & $9,30 \pm 0,37 \mathrm{a}$ & $7,91 \pm 0,26 \mathrm{~A}$ & $1,85 \pm 0,07$ & $2,02 \pm 0,07$ & $1,93 \pm 0,05$ & $1,57 \pm 0,06$ & $1,70 \pm 0,06$ & $1,63 \pm 0,04$ \\
\hline $5 / 8 \mathrm{Ne} 3 / 8 \mathrm{Ch}$ & $6,53 \pm 0,37 b$ & $7,51 \pm 0,37 b$ & $7,02 \pm 0,26 \mathrm{~B}$ & $1,84 \pm 0,07$ & $1,88 \pm 0,07$ & $1,86 \pm 0,05$ & $1,60 \pm 0,06$ & $1,59 \pm 0,06$ & $1,60 \pm 0,04$ \\
\hline \multirow[t]{2}{*}{ Média } & $6,53 \pm 0,26$ & $8,40 \pm 0,26$ & & $1,85 \pm 0,05$ & $1,94 \pm 0,05$ & & $1,58 \pm 0,04$ & $1,65 \pm 0,04$ & \\
\hline & \multicolumn{2}{|c|}{ Omaso, $\mathrm{kg}$} & & \multicolumn{2}{|c|}{ Omaso, \% PCVZ } & & \multicolumn{2}{|c|}{ Omaso, \% PAB } & \\
\hline $5 / 8 \mathrm{Ch} 3 / 8 \mathrm{Ne}$ & $3,27 \pm 0,37$ & $4,83 \pm 0,37$ & $4,05 \pm 0,26$ & $0,93 \pm 0,09$ & $1,04 \pm 0,09$ & $0,99 \pm 0,07$ & $0,79 \pm 0,09$ & $0,88 \pm 0,09$ & $0,83 \pm 0,06$ \\
\hline $5 / 8 \mathrm{Ne} 3 / 8 \mathrm{Ch}$ & $3,49 \pm 0,37$ & $3,85 \pm 0,37$ & $3,67 \pm 0,26$ & $0,99 \pm 0,09$ & $0,96 \pm 0,09$ & $0,98 \pm 0,07$ & $0,87 \pm 0,09$ & $0,81 \pm 0,09$ & $0,84 \pm 0,06$ \\
\hline \multirow[t]{2}{*}{ Média } & $3,38 \pm 0,26 \mathrm{D}$ & $4,34 \pm 0,26 \mathrm{C}$ & & $0,96 \pm 0,07$ & $1,00 \pm 0,07$ & & $0,83 \pm 0,06$ & $0,84 \pm 0,06$ & \\
\hline & \multicolumn{2}{|c|}{ Abomaso, $\mathrm{kg}$} & & \multicolumn{2}{|c|}{ Abomaso, \% PCVZ } & & \multicolumn{2}{|c|}{ Abomaso, $\%$ PAB } & \\
\hline $5 / 8 \mathrm{Ch} 3 / 8 \mathrm{Ne}$ & $1,85 \pm 0,15 \mathrm{c}$ & $2,88 \pm 0,15 \mathrm{a}$ & $2,36 \pm 0,10$ & $0,52 \pm 0,03$ & $0,63 \pm 0,03$ & $0,57 \pm 0,02$ & $0,44 \pm 0,03$ & $0,53 \pm 0,03$ & $0,49 \pm 0,02$ \\
\hline $5 / 8 \mathrm{Ne} 3 / 8 \mathrm{Ch}$ & $1,97 \pm 0,15 \mathrm{bc}$ & $2,32 \pm 0,15 b$ & $2,15 \pm 0,10$ & $0,56 \pm 0,03$ & $0,57 \pm 0,03$ & $0,57 \pm 0,02$ & $0,49 \pm 0,03$ & $0,49 \pm 0,03$ & $0,49 \pm 0,02$ \\
\hline \multirow[t]{2}{*}{ Média } & $1,91 \pm 0,10 \mathrm{C}$ & $2,60 \pm 0,10 \mathrm{D}$ & & $0,54 \pm 0,02$ & $0,60 \pm 0,02$ & & $0,47 \pm 0,02$ & $0,51 \pm 0,02$ & \\
\hline & \multicolumn{2}{|c|}{ Intestinos, $\mathrm{kg}$} & & \multicolumn{2}{|c|}{ Intestinos, \% PCVZ } & & \multicolumn{2}{|c|}{ Intestinos, $\%$ PAB } & \\
\hline $5 / 8 \mathrm{Ch} 3 / 8 \mathrm{Ne}$ & $7,29 \pm 0,58 \mathrm{ab}$ & $8,89 \pm 0,58 \mathrm{a}$ & $8,09 \pm 0,41 \mathrm{~A}$ & $2,07 \pm 0,14$ & $1,92 \pm 0,14$ & $2,00 \pm 0,10$ & $1,75 \pm 0,12$ & $1,63 \pm 0,12$ & $1,69 \pm 0,08$ \\
\hline $5 / 8 \mathrm{Ne} 3 / 8 \mathrm{Ch}$ & $7,16 \pm 0,58 b$ & $6,08 \pm 0,58 b$ & $6,62 \pm 0,41 \mathrm{~B}$ & $2,02 \pm 0,14$ & $1,52 \pm 0,14$ & $1,77 \pm 0,10$ & $1,75 \pm 0,12$ & $1,29 \pm 0,12$ & $1,52 \pm 0,08$ \\
\hline Média & $7,22 \pm 0,41$ & $7,49 \pm 0,41$ & & $2,04 \pm 0,10 \mathrm{C}$ & $1,73 \pm 0,10 \mathrm{D}$ & & $1,75 \pm 0,08 \mathrm{C}$ & $1,46 \pm 0,08 \mathrm{D}$ & \\
\hline
\end{tabular}


Tabela 4 - Total de órgãos internos, total do trato digestório e sangue em peso absoluto e em relação aos pesos de corpo vazio (PCVZ) e de abate (PAB) de acordo com a categoria e grupo genético

\begin{tabular}{|c|c|c|c|c|c|c|c|c|c|}
\hline \multirow{3}{*}{ Grupo genético } & \multicolumn{2}{|c|}{ Categoria } & \multirow{3}{*}{ Média } & \multicolumn{2}{|c|}{ Categoria } & \multirow{3}{*}{ Média } & \multicolumn{2}{|c|}{ Categoria } & \multirow{3}{*}{ Média } \\
\hline & Novilho & Vaca & & Novilho & Vaca & & Novilho & Vaca & \\
\hline & \multicolumn{2}{|c|}{ Órgãos internos, $\mathrm{kg}$} & & \multicolumn{2}{|c|}{ Órgãos internos, \% PCVZ } & & \multicolumn{2}{|c|}{ Órgãos internos, \% PAB } & \\
\hline $5 / 8 \mathrm{Ch} 3 / 8 \mathrm{Ne}$ & $13,9 \pm 0,67$ & $16,1 \pm 0,67$ & $15,0 \pm 0,48$ & $3,94 \pm 0,13$ & $3,53 \pm 0,13$ & $3,73 \pm 0,09$ & $3,33 \pm 0,11$ & $2,97 \pm 0,11$ & $3,14 \pm 0,08$ \\
\hline $5 / 8 \mathrm{Ne} 3 / 8 \mathrm{Ch}$ & $13,9 \pm 0,67$ & $14,9 \pm 0,67$ & $14,4 \pm 0,48$ & $3,90 \pm 0,13$ & $3,74 \pm 0,13$ & $3,82 \pm 0,09$ & $3,38 \pm 0,11$ & $3,15 \pm 0,11$ & $3,27 \pm 0,08$ \\
\hline \multirow[t]{2}{*}{ Média } & $13,9 \pm 0,48 \mathrm{D}$ & $15,5 \pm 0,48 \mathrm{C}$ & & $3,92 \pm 0,09 \mathrm{C}$ & $3,63 \pm 0,09 \mathrm{D}$ & & $3,35 \pm 0,08 \mathrm{C}$ & $3,06 \pm 0,08 \mathrm{D}$ & \\
\hline & \multicolumn{2}{|c|}{ Trato digestório, $\mathrm{kg}$} & & \multicolumn{2}{|c|}{ Trato digestório, \% PCVZ } & & \multicolumn{2}{|c|}{ Trato digestório, \% PAB } & \\
\hline $5 / 8 \mathrm{Ch} 3 / 8 \mathrm{Ne}$ & $18,9 \pm 1,08 \mathrm{~b}$ & $25,9 \pm 1,08 \mathrm{a}$ & $22,4 \pm 0,76 \mathrm{~A}$ & $5,39 \pm 0,23$ & $5,64 \pm 0,23$ & $5,51 \pm 0,16$ & $4,55 \pm 0,20$ & $4,73 \pm 0,20$ & $4,64 \pm 0,14$ \\
\hline $5 / 8 \mathrm{Ne} 3 / 8 \mathrm{Ch}$ & $19,1 \pm 1,08 \mathrm{~b}$ & $19,8 \pm 1,08 \mathrm{~b}$ & $19,4 \pm 0,76 \mathrm{~B}$ & $5,42 \pm 0,23$ & $4,96 \pm 0,23$ & $5,19 \pm 0,16$ & $4,70 \pm 0,20$ & $4,18 \pm 0,20$ & $4,44 \pm 0,14$ \\
\hline \multirow[t]{2}{*}{ Média } & $19,0 \pm 0,76 \mathrm{D}$ & $22,9 \pm 0,76 \mathrm{C}$ & & $5,40 \pm 0,16$ & $5,30 \pm 0,16$ & & $4,62 \pm 0,14$ & $4,46 \pm 0,14$ & \\
\hline & \multicolumn{2}{|c|}{ Sangue, $\mathrm{kg}$} & & \multicolumn{2}{|c|}{ Sangue, \% PCVZ } & & \multicolumn{2}{|c|}{ Sangue, $\%$ PAB } & \\
\hline $5 / 8 \mathrm{Ch} 3 / 8 \mathrm{Ne}$ & $11,26 \pm 1,08$ & $14,03 \pm 1,08$ & $12,65 \pm 0,76$ & $3,17 \pm 0,19$ & $3,05 \pm 0,19$ & $3,11 \pm 0,13$ & $2,67 \pm 0,17$ & $2,56 \pm 0,17$ & $2,61 \pm 0,12$ \\
\hline $5 / 8 \mathrm{Ne} 3 / 8 \mathrm{Ch}$ & $10,83 \pm 1,08$ & $12,06 \pm 1,08$ & $11,45 \pm 0,76$ & $3,04 \pm 0,19$ & $2,98 \pm 0,19$ & $3,02 \pm 0,13$ & $2,64 \pm 0,17$ & $2,53 \pm 0,17$ & $2,58 \pm 0,12$ \\
\hline Média & $11,05 \pm 0,76 \mathrm{D}$ & $13,05 \pm 0,76 \mathrm{C}$ & & $3,11 \pm 0,13$ & $3,02 \pm 0,13$ & & $2,66 \pm 0,12$ & $2,54 \pm 0,12$ & \\
\hline
\end{tabular}

Houve interação $(\mathrm{P}<0,05)$ para $\mathrm{o}$ peso absoluto do abomaso, sendo que as vacas $5 / 8 \mathrm{Ch}$ $3 / 8 \mathrm{Ne}$ foram superiores aos novilhos do mesmo grupo genético, enquanto que não houve diferença entre as categorias nos animais $5 / 8 \mathrm{Ne} 3 / 8 \mathrm{Ch}$. PACHECO et al. (2005) também encontraram interação entre grupo genético e categoria, atribuindo tal fato ao tipo de seleção de nutrientes que é feito pelos animais, mesmo com igualdade na relação volumoso:concentrado na dieta. FERREIRA et al. (2000) e GESUALDI JÚNIOR et al. (2001) verificaram aumento no peso do abomaso com aumento do nível de concentrado na dieta. De acordo com FERREL et al. (1976), o abomaso participa ativamente do processo de digestão e absorção, podendo ser maior em animais que recebem maior fração de concentrado na dieta.

Interação $(\mathrm{P}<0,05)$ também foi encontrada para o peso absoluto dos intestinos, encontrando a mesma variação que ocorreu no peso do abomaso, com maior valor para as vacas $5 / 8 \mathrm{Ch} 3 / 8 \mathrm{Ne}$. Resultado idêntico foi encontrado por PACHECO et al. (2005), sendo que na categoria de maior idade (jovem) os animais $5 / 8 \mathrm{Ch} 3 / 8 \mathrm{Ne}$ foram superiores aos $5 / 8 \mathrm{Ne} 3 / 8 \mathrm{Ch}$ no peso dos intestinos, porém não houve diferença entre os grupos genéticos na categoria superjovem.

As vacas apresentaram superioridade $(\mathrm{P}<0,05)$ no peso absoluto do total dos órgãos internos (Tabela 4), influenciado pelos maiores pesos de coração e fígado, visto que a correlação desses órgãos com o total de órgãos internos foi de 0,76 e
0,84, respectivamente $(\mathrm{P}<0,0001)$. Quando comparados em termos relativos, o peso total de órgãos vitais dos novilhos foram superiores ao das vacas. Isso ocorre porque os órgãos vitais apresentam maior desenvolvimento em fase mais precoce devido à hiperplasia, que é o aumento no número de células dos tecidos, mais intensa em estádios iniciais da vida dos animais (GESUALDI JÚNIOR et al., 2001). Avaliando-se o total de órgãos internos com base nos grupos genéticos, observa-se que não houve diferença significativa. MENEZES et al. (2007) observaram maior peso do total de órgãos internos para os animais Charolês em relação aos Nelore, responsabilizando a maior seleção pela parte concentrada da dieta por este fato.

Em valores absolutos, o peso total do trato digestório apresentou interação $(\mathrm{P}<0,05)$ entre a categoria e o grupo genético, com superioridade para as vacas no grupo genético $5 / 8 \mathrm{Ch} 3 / 8 \mathrm{Ne}$ e similaridade entre as categorias nos animais de genótipo $5 / 8 \mathrm{Ne} 3 / 8 \mathrm{Ch}, \quad$ acompanhando o comportamento apresentado para alguns compartimentos do trato gastrintestinal quando avaliados de maneira isolada (Tabela 3). Entre as categorias de bovinos, as vacas apresentaram maior peso do total de trato digestório, o que está relacionado ao maior peso de abate das fêmeas de descarte. MISSIO et al. (2013) verificaram que o incremento no peso de abate de novilhos promoveu aumento linear no peso do trato digestório. No presente estudo o peso de abate esteve positiva e significativamente associado ao peso do trato 
digestório $(\mathrm{r}=0,80 ; \mathrm{P}<0,0001)$. Outro fator que auxilia a explicação do maior peso do trato digestório é a manifestação do ganho compensatório nas vacas, pois, segundo HOGG (1991), esse ganho promove aumento no consumo de alimentos e, consequentemente, no tamanho do trato gastrintestinal.

Quando o peso absoluto do trato digestório é analisado de acordo com o grupo genético estudado, verifica-se que os animais $5 / 8 \mathrm{Ch} 3 / 8 \mathrm{Ne}$ foram $15,4 \%$ superiores aos bovinos $5 / 8 \mathrm{Ne} 3 / 8 \mathrm{Ch}$. Resultados similares foram reportados por FERREL \& JENKINS (1998), que constataram maior peso do trato gastrintestinal em animais das raças Angus e Hereford em comparação aos da raça Brahman. Esses autores associaram esse resultado ao menor potencial de consumo das raças zebuínas em relação às taurinas. PERIPOLLI et al. (2013) também observaram menores pesos do trato digestório em animais zebuínos (Brahman) em relação a novilhos da raça Brangus e Hereford terminados em confinamento. Porém quando essa variável é apresentada em relação aos pesos de corpo vazio e de abate, não há diferença entre os genótipos. No estudo de MENEZES et al. (2007), o peso do trato gastrintestinal afetou o rendimento de carcaça, sendo que a correlação entre essas duas variáveis foi de 0,61 ( $\mathrm{P}<0,01)$; entretanto, no presente estudo a correlação entre essas variáveis foi baixa e não significativa $(r=-0,08 ; P=0,7088)$.

As fêmeas de descarte apresentaram maior peso de sangue, 13,05 contra $11,05 \mathrm{~kg}$, o que deve estar associado à superioridade no peso dos conjuntos de órgãos internos e do trato digestório. RIBEIRO et al. (2001) citam que o aumento no volume de sangue acompanha o incremento dos órgãos vitais e do trato digestório, o que seria necessário para acompanhar variações na taxa metabólica dos animais. No presente estudo, o peso de sangue esteve positivamente associado com o conjunto de órgãos internos $(\mathrm{r}=0,70 ; \mathrm{P}=0,0001) \mathrm{e}$ do trato digestório $(\mathrm{r}=0,67 ; \mathrm{P}=0,0003)$.

\section{CONCLUSÕES}

Vacas de descarte apresentam maior massa dos órgãos internos e do trato digestório que novilhos abatidos aos 24 meses, enquanto bovinos do grupo genético $5 / 8 \mathrm{Ch} 3 / 8 \mathrm{Ne}$ apresentam maior peso de compartimentos e do total do trato digestório em relação a bovinos $5 / 8 \mathrm{Ne} 3 / 8 \mathrm{Ch}$.

\section{REFERÊNCIAS}

ANUALPEC 2010. Anuário da pecuária brasileira
FNP. São Paulo: Prol Editora Gráfica, 2010. 360p.

CATTELAN, J.; MENEZES, L.F.G.; FERREIRA, J.J.; RESTLE, J.; ALVES FILHO, D.C., BRONDANI, I.L. Gorduras de descarte e componentes externos do corpo de novilhos e vacas de descarte de diferentes grupos genéticos. Ciência Rural, v.40, n.12, p.2541-2548, 2010.

CATTON, J.S.; DHUYVETTER, D.V. Influence of energy supplementation on grazing ruminants: requirements and responses. Journal of Animal Science, v.75, n.4, p.533-542, 1997.

FERREIRA, M.A.; VALADARES FILHO, S.C.; MUNIZ, E.B.; VERAS, A.S.C. Características das carcaças, biometria do trato gastrintestinal, tamanho dos órgãos internos e conteúdo gastrintestinal de bovinos $\mathrm{F}_{1}$ Simental x Nelore alimentados com dietas contendo vários níveis de concentrado. Revista Brasileira de Zootecnia, v.29, n.4, p.1174-1182, 2000.

FERREL, C.L.; GARRET, W.N.; HINMAN, N. Estimation of body composition in pregnant and non pregnant heifers. Journal of Animal Science, v.42, p.1158-1166, 1976.

FERREL, C.L.; JENKINS, T.G. Body composition and energy utilization by steers of diverse genotypes fed a high-concentrate diet during the finishing period: II Angus, Boran, Brahman, Hereford, and Tuli Sires. Journal of Animal Science, v.76, p.647-657, 1998.

GESUALDI JÚNIOR, R.A., VELOSO, C.M.; PAULINO, M.F.; VALADARES FILHO, S.C.; GESUALDI, A.C.L.S.; CECON, P.R. Níveis de concentrado na dieta de bovinos $F_{1}$ Limousin $x$ Nelore: peso dos órgãos internos e trato digestivo. Revista Brasileira de Zootecnia, v.30, n.6, p.1866-1971, 2001.

HOGG, B.W. Compensatory growth in ruminants. In: PEARSON, A.M., DUTSON, T.R. (Eds.). Growth regulation in farm animals. London: Elsevier Applied Science. 1991. p.103-134.

JOHNSON, D.E.; JOHNSON, K.A.; BALDWIN, R.L. Changes in liver and gastrointestinal tract energy demands in response to physiological workload in ruminants. Journal of Nutrition, v.120, p.649-655, 1990.

JORGE, A.M.; FONTES, C.A.A. Desenvolvimento relativo das partes do corpo de zebuínos de quatro raças. Ciência Rural, v.31, n.5, p.857-861, 2001.

KUSS, F.; RESTLE, J.; BRONDANI, I.L.; PASCOAL, L.L.; MENEZES, L.F.G.; LEITE, D.T.; SANTOS, M.F. Componentes externos do corpo e gordura de descarte em vacas mestiças Charolês x Nelore abatidas com diferentes pesos. Revista Brasileira de Zootecnia, v.36, n.4, p.865873, 2007.

KUSS, F.; BARCELLOS, J.O.J.; LÓPEZ, J.; RESTLE, J.; MOLETTA, J.L.; PAULA, M.C. Componentes nãointegrantes da carcaça de novilhos não-castrados terminados em confinamento e abatidos aos 16 ou 26 meses de idade. Revista Brasileira de Zootecnia, v.37, n.10, p.1829-1836, 2009. 
LOWAN, B.G.; SCOTT, N.; SOMERVILLE, S. D.A.O.; LAMPERT, V.N.; VELHO, M.M.S. Condition scoring beef cattle. Edinburgh: East of Componentes não-integrantes da carcaça de bovinos de Scotland College of Agriculture. 1973. 8p. (Bulletin 6)

MENEZES, L.F.G.; RESTLE, J.; BRONDANI, I.L.; KUSS, F.; ALVES FILHO, D.C.; SILVEIRA, M.F.; LEITE, D.T. Órgãos internos e trato gastrintestinal de novilhos de gerações avançadas do cruzamento rotativo entre as raças Charolês e Nelore, terminados em confinamento. Revista Brasileira Zootecnia, v.36, n.1, p.120-129, 2007.

MISSIO, R.L.; RESTLE, J.; MOLETTA, J.L.; KUSS, F.; NEIVA, J.N.M.; MIOTTO, F.R.C.; PRADO, I.N.; ELEJALDE, D.A.G.; PEROTTO, D. Componentes do corpo vazio de vacas da raça Purunã abatidas com pesos distintos. Semina: Ciências Agrárias, v.34, n.2, p.83894, 2013.

NATIONAL RESEARCH COUNCIL - NRC. Nutrient requirements of domestic animals. 7 th revisededition. Washington: National Academy Press, 2001. 242p.

OWENS, F.N.; GILL, D.R.; SECRIST, D.S.; COLEMAN, S.W. Review of some aspects of growth and development of feedlot cattle. Journal of Animal Science, v.73, p.3152-3172, 1995.

PACHECO, P.S.; RESTLE, J.; SILVA, J.H.S.; ARBOITTE, M.Z.; ALVES FILHO, D.C.; FREITAS, A.K.; ROSA, J.R.P.; PÁDUA, J.T. Características das partes do corpo não-integrantes da carcaça de novilhos jovens e superjovens de diferentes grupos genéticos. Revista Brasileira de Zootecnia, v.34, n.5, p.1678-1690, 2005.

PERIPOLLI, V.; BARCELLOS, J.O.J; OLMEDO, três grupos genéticos terminados em confinamento ou pastejo rotacionado com suplementação. Revista Brasileira de Saúde e Produção Animal, v.14, n.1, p.209-223, 2013.

RIBEIRO, T.R.; PEREIRA, J.C.; LEÃO, M.I.; OLIVEIRA, M.V.M.; QUEIROZ, A.C.; CECON, P.R.; MELO, R.C.A.Tamanho dos órgãos e vísceras de bezerros holandeses, para a produção de vitelos recebendo dietas com diferentes níveis de concentrado. Revista Brasileira de Zootecnia, v.30, n.6, p.2163-2168, 2001. (Suplemento)

STATISTICAL ANALISYS SYSTEM - SAS. SAS Language Reference.Version 6. Cary, NC: SAS institute, 2001 ,

SILVA, F.F.; VALADARES FILHO, S.C.; ÍTAVO, L.C.V.; VELOSO, C.M.; PAULINO, M.F.; VALADARES, R.F.D.; SILVA, P.A.; GALVÃO, R.M. Consumo, desempenho, características de carcaça, e biometria do trato gastrintestinal e dos órgãos internos de novilhos Nelore recebendo diferentes níveis de concentrado e proteína. Revista Brasileira de Zootecnia, v.31, n.4, p.1849-1864, 2002.

VAN SOEST, P. J. Nutritional ecology of the ruminant. 2. ed. Ithaca: Comstock Publishing Associates, 1994. 476 p.

VAZ, F.N.; RESTLE, J.; ARBOITTE, M.Z.; PASCOAL, L.L.; FATURI, C.; JONER, G. Fatores relacionados ao rendimento de carcaça de novilhos ou novilhas superjovens, terminados em pastagem cultivada. Ciência Animal Brasileira, v. 11, n. 1, p. 53-61, 2010.

Protocolado em: 05 jun. 2013 Aceito em: 05 set. 2013. 